Polymer Journal, Vol. 22, No. 10, pp 909—918 (1990)

\title{
X-Ray Diffraction Study of Polyimide Blends Compatibility
}

\author{
Jwo-Huei Jou and Peir-Teh Huang \\ Department of Materials Science and Engineering, Tsing Hua University, \\ Hsin Chu 30043, Taiwan, R.O.C.
}

(Received February 17, 1990)

\begin{abstract}
An X-ray diffractometry method has been employed to investigate the compatibility characteristics of two polyimide blend systems. From the in-plane and out-of-plane $\mathrm{X}$-ray diffraction patterns, it is learned that the higher in chain rigidity, the higher ordering and film orientation can one observe in the on-substrate cured polyimide films. By comparing the out-of-plane diffraction patterns from the stacked films of the blends, laminates, random copolymers, and alternating copolymers, it can be concluded that the blends of PMDA-ODA/PMDA-PDA (pyromellitic dianhydride-oxydianiline/pyromellitic dianhydride-p-phenylenediamine) are compatible at all compositions except at above $90 \%$ PMDA-PDA. The blends of PMDA-ODA/PMDA-B (pyromellitic dianhydride-benzidine) are not compatible. Kept mixed longer, the characteristic diffraction peak intensities of the blends with PMDA-B composition less than $50 \%$ have decreased and become undistinguishable from those of the corresponding random copolymers, and those with higher PMDA-B composition have also decreased significantly. It is plausible to say that the blends with diffraction patterns undistinguishable from those of the corresponding random copolymers have actually converted to random copolymers due to exchange reactions occurred among the amic acid compounds when in the solution state.
\end{abstract}

KEY WORDS Polyimides / Blend / Compatibility / X-Ray Diffraction /

Polyimides have been extensively studied for their important application in electronic packaging. ${ }^{1-6}$ Generally, they exhibit relatively low dielectric constant, high glass transition and low thermal expansion. These properties, especially the latter one, as well as mechanical properties strongly depend on the rigidity of chain structure. Those with rigid rod-like chain structure have relatively low thermal expansion coefficients and high tensile moduli, but weak in elongation and much brittle. The ones with semi-flexible chain structure exhibit better mechanical behaviors, but have undesired high thermal expansion coefficients. The shortcomings exhibited in these two types of polymers may be resolved at once by direct blending of their precursor amic acid solutions. The addition of a small portion of a semi-flexible polyimide can greatly improve the toughness of a given brittle polyimide. ${ }^{7}$ Thermal expansion coefficient can also be tailored conveniently according to its composition.

However, in the study, ${ }^{7}$ it is shown that the characteristic curves of the tensile moduli with varying compositions are very different for two different blend systems. It has brought to our attention naturally that compatibility may be the origin of the difference. Therefore, the major purpose of this study is to further understand the compatibilities of the blend systems.

In this study, two blend systems, one compatible and one incompatible, have been investigated. Each system consists of a semi-flexible polyimide and a rigid polyimide. These were obtained by mixing their precursor solutions, followed by a thermal curing. The effect of composition has also been examined.

Furthermore, it has been reported ${ }^{8-11}$ that 
polyamic acids, before cured to solid imides, would undergo exchange reactions with other amic acid compounds in the solution state. If this phenomenon happens in a compatible binary mixture, copolyamic acids will be resulted immediately. With time, these copolymers may become random copolymers since exchange reactions may take place at any reactive sites randomly. Even for an incompatible binary mixture, as long as it is in solution and before solidified, exchange reactions will be likely to occur, though may be slowly. If kept mixed for a much longer time period, partial phase mixing or even random copolymers, eventually, can be obtained through such a randomization due to exchange reactions. To investigate such an effect owing to exchange reactions, specimens with different mixing time have been prepared.

As presented by Feger ${ }^{9}$ and Volksen et al. ${ }^{12}$ DMTA (dynamic mechanical thermal analyser) can be used to investigate the compatibility of polyimide blends. This is true only if the studied polyimides have very different mechanical behaviors. Especially, the glass transition temperatures must be well separated. Unfortunately, most polyimides do not exhibit glass transition at temperature below $400^{\circ} \mathrm{C}$. Though they may exhibit at above $400^{\circ} \mathrm{C}$, most of the glass transition temperatures are not so well separated. Besides, restriction of DMTA of not running above $500^{\circ} \mathrm{C}$ limits such studies. One would not encounter such an inconvenience if using X-ray diffractometry. ${ }^{13,14}$

From X-ray diffraction patterns, one can easily tell whether the blend systems are compatible or not. Though, it will be even more straightforward if some references can be prepared for comparison. In this study, it has been so done by preparing three different kinds of specimens. There are (1) laminates composed of alternating this layers of the two studied polyimides, (2) random copolymers, and (3) alternating copolymers. The laminates are used to represent completely phase segregation. If the polyblends are completely incompatible, their diffraction patterns should be, presumably, similar to those of the laminates. If the diffraction patterns of the blends are similar to those of the random copolymers, it seems plausible to say that the above-mentioned exchange reaction may have taken place and the resulted mixtures have become random copolymers. As to a third reference, it would be much better to prepare some block copolymers, if doable. Howeve, in this study, block copolyamic acids could not be made though using the synthesis method proposed in the papers. ${ }^{18-19}$ By using the method, the polymers prepared look much like alternating, instead of block, copolyamic acids according to their X-ray diffraction patterns. This will be further discussed in a latter section. Also, according to the related formulae given in the same papers, one can easily tell that the resulted copolymers are supposed to be alternative, not block. Though, alternating copolymers have been chosen as a third reference. In the following section, experimental procedures of preparing these samples are described.

\section{EXPERIMENTAL}

\section{Materials}

The starting materials used in this study are pyromellitic dianhydride (PMDA) and diamines: 4,4'-oxydianiline (ODA), p-phenylenediamine (PDA) and benzidine (B). The solvent used is $N$-Methyl pyrrolidone (NMP). These materials were used as received.

\section{Polycondensation of Polyamic acids}

A. Homopolymers. Homopolymers of PMDA-ODA, PMDA-PDA, and PMDA-B polyamic acids were prepared as follows ${ }^{15}$ : In a four-neck round bottle flask, dissolving diamine, ODA, PDA or B, in the NMP solvent. When the diamine was completely dissolved, equal-molar of dianhydride PMDA was added gradually. The reaction had proceeded for eight hours with stirring. The entire process was done in a nitrogen atmosphere. The resulted 


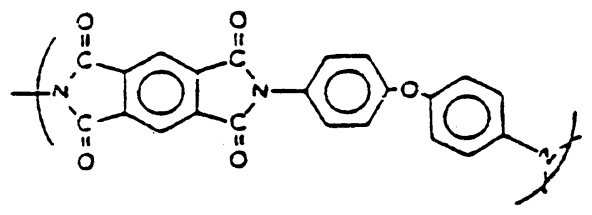

PMDA-ODA

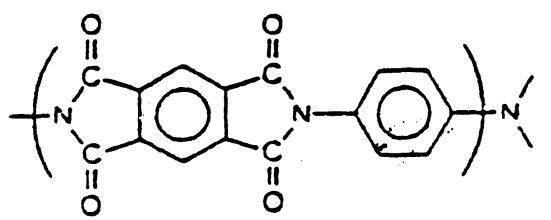

PMDA-PDA

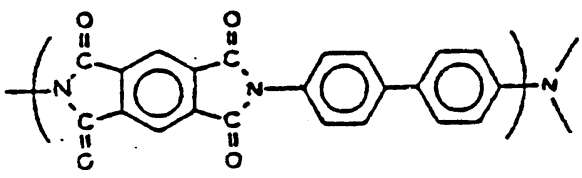

PMDA-B

Figure 1. Molecular structures of polyimides PMDAODA, PMDA-PDA, and PMDA-B.

solutions have a solid content of $14 \mathrm{wt} \%$. The molecular structures of the resulted polyamic acids after cured to solid imides are shown in Figure 1.

B. Polyblends. Blends of PMDA-ODA with PMDA-PDA, and PMDA-ODA with PMDA-B were obtained by mixing the resulted polyamic acids. The mixing was done under nitrogen for five minutes. The resulted, mixed solutions were then kept frozen before use. Another batchs were prepared in the same way, but kept at room temperature for one or two days before use.

C. Random Copolymers. Random copolyamic acids of PMDA-ODA/PMDA-PDA and PMDA-ODA/PMDA-B were prepared, ac- cording to the method reported in the references. ${ }^{16,17}$ Similarly, two different diamines, ODA/PDA or ODA/B, were together dissolved in the NMP solvent before the addition of PMDA. Four different compositions for each copolyamic acid were prepared.

D. Alternating Copolymers. Alternating copolyamic acids of PMDA-ODA/PMDAPDA and PMDA-ODA/PMDA-B were prepared by using the method reported in the papers. ${ }^{18,19}$ (As mentioned earlier, according to their formulae, the method should be, supposedly, for preparaing alternating copolymers. This is very clear especially when the molar ratio of the two diamines is $1: 1$.) This was done by adding an excess amount of PMDA into one of the two diamines, which was first dissolved. After five hours of reaction, the other diamine was added and the reaction continued for another five hours. Again, several different compositions for each alternating copolyamic acid were prepared.

\section{Imidization}

Solid films of the above-mentioned polyamic acids were prepared by spin-casting the solutions on glass substrates, followed by prebacking at $80^{\circ} \mathrm{C}$ for an half hour, and then cured from $80^{\circ} \mathrm{C}$ to $400^{\circ} \mathrm{C}$ in three hours. Though polyimide films of PMDA-ODA could be made at a higher ramp rate, those of PMDA-PDA and PMDA-B would be much less fragile only if cured slowly. Therefore, the ramp rate was kept as low as above described. Since most polyamic acids would be nearly fully imidized at temperatures higher than $350^{\circ} \mathrm{C}$ in $30 \mathrm{~min}$, and varying degrees of imidization have a significant effect on polyimide chain ordering, ${ }^{20}$ curing to $400^{\circ} \mathrm{C}$ seems adequate.

\section{X-Ray Specimen Preparation}

The resulted polyimide films are about $20 \mu \mathrm{m}$ thick. In a previous X-ray experiment, it is found that these polyimide films must be thicker than $200 \mu \mathrm{m}$ so that the X-ray will not penetrate through the films. In order to 
compare quantitatively, relatively thick samples are therefore required. This was done by stacking many pieces, such as fifty or so, of the films together. By so doing, one has one other advantage that not only structure information in the out-of-plane (film thickness) direction, but also in the in-plane (film plane) direction can be obtained.

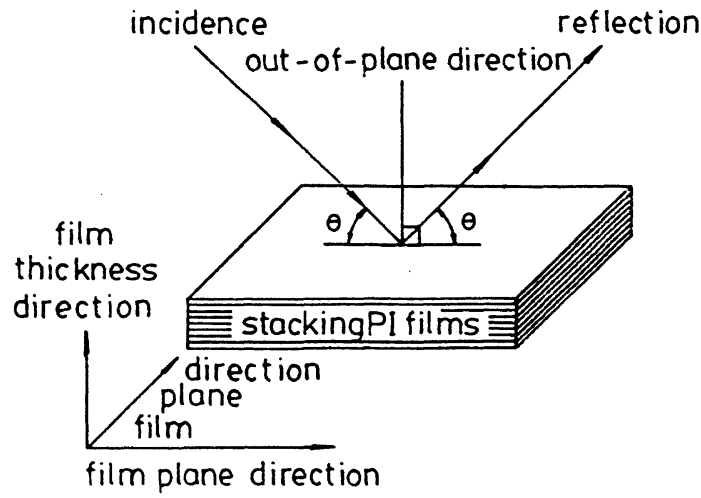

(a)

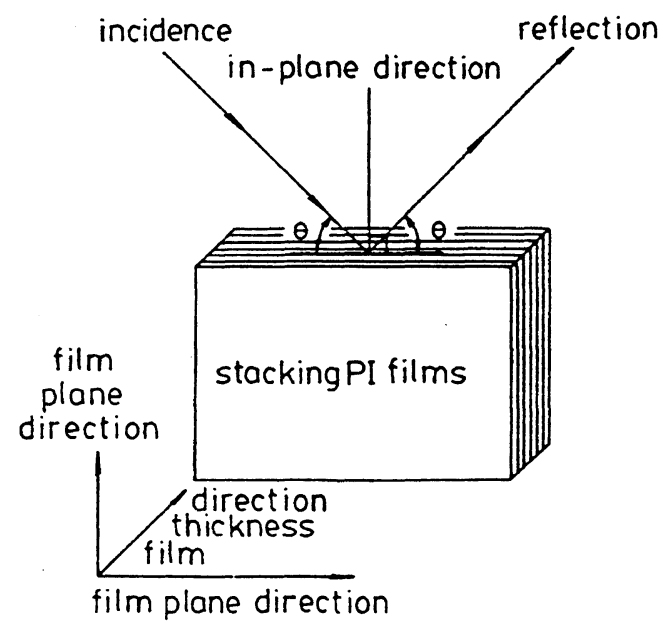

(b)

Figure 2. Schematic illustration of the arrangements of the X-ray diffraction experiments: (a) the out-of-plane diffraction, in which the sample is so arranged that its film plane is perpendicular to the plane formed by the incident and reflected beams, and (b) the in-plane diffraction, in which its film plane is parallel to the plane formed by the incident and reflected beams.

\section{$X$-Ray Experiment}

$\mathrm{X}$-ray experiments were done using a Rigaku Wide-Angle X-Ray Diffractometer with a nickel-filtered copper $K_{\alpha 1}$-radiation. Its power setting was at $40 \mathrm{kV}$ and $20 \mathrm{~mA}$. The line-focus slot has a dimension of $8 \mathrm{~mm} \times 0.04 \mathrm{~mm}$. The arrangement of $\mathrm{X}$-ray diffraction experiment is illustrated schematically in Figure 2. For out-of-plane diffraction, the stacked films were so arranged that the film plane was perpendicular to the plane formed by the incident and reflected X-ray beams, as shown in Figure 2(a). For in-plane diffraction, the samples were so arranged that the film plane was parallel to the plane formed by the incident and reflected $\mathrm{X}$-ray beams, as shown in Figure 2(b). Since the lengths of the repeating units of these polyimides can be seen much larger than the intermolecular spacings, ${ }^{21,22}$ diffraction peaks, if any, that correspond to the repeating units, are expected to appear at small diffraction angle, relatively speaking. Diffraction peaks that correspond to the intermolecular spacings should appear at large diffraction angle.

\section{RESULTS AND DISCUSSION}

\section{Ordering and Orientation}

Figure 3 shows the X-ray diffraction patterns of PMDA-PDA. As shown in the out-of-lane diffraction pattern, (a), at 20.8 degrees, there

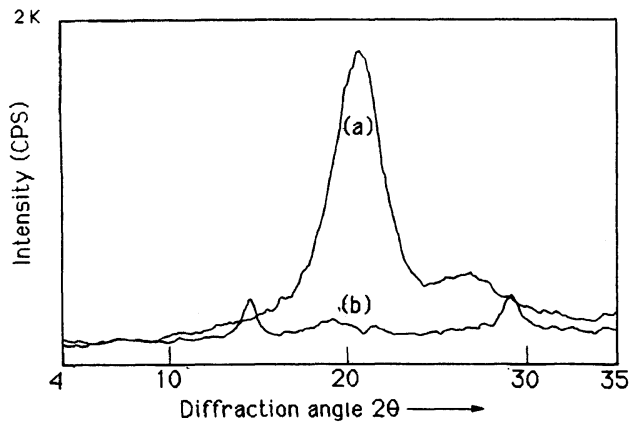

Figure 3. X-Ray diffraction patterns from (a) the out-of-plane (film thickness), and (b) the in-plane (film plane) directions of the stacked PMDA-PDA polyimide films. 
is a sharp peak, which corresponds to the intermolecular spacing of the imide chains of PMDA-PDA. ${ }^{21,22}$ In the in-plane diffraction pattern, (b), there is nearly no peak at the same angle. These results indicate not only that the on substrate cured films of PMDA-PDA are very anisotropic, structurewise, but also that the imide chains are predominantly aligned in the plane of the films. The resulted films seem have been highly oriented. Such a high film orientation is typical for polyimides having a very symmetrical, rigid chain structure when cured on substrate. As calculated from the peak position, its intermolecular distance is $\mathbf{0 . 4 3 0}$ nm.

Figure 4 shows the $\mathrm{X}$-ray diffraction patterns of PMDA-B. As can be seen, PMDA-B also

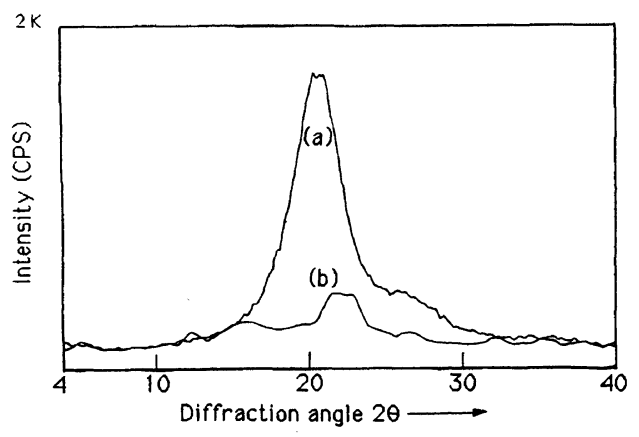

Figure 4. X-Ray diffraction patterns from (a) the out-of-plane (film thickness), and (b) the in-plane (film plane) directions of the stacked PMDA-B polyimide films.

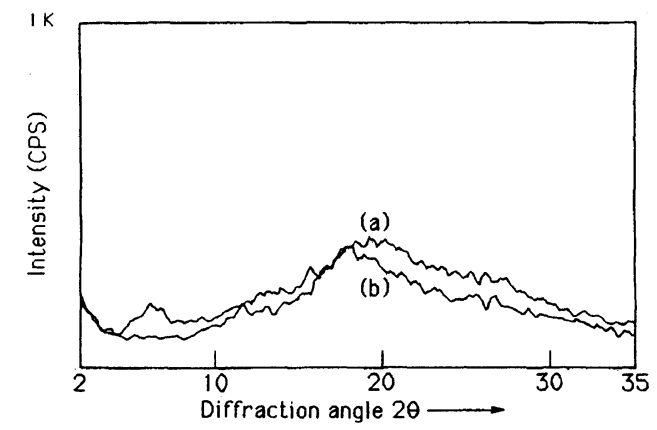

Figure 5. X-Ray diffraction patterns from (a) the out-of-plane (film thickness), and (b) the in-plane (film plane) directions of the stacked PMDA-ODA polyimide films. exhibits very high film orientation. Its intermolecular distance is $0.427 \mathrm{~nm}$, very close to that of PMDA-PDA. This is quite reasonable since these two polyimides have similar symmetrical, planar chain structures.

However, PMDA-ODA exhibits much less in molecular ordering and in film orientation, as shown in Figure 5. In PMDA-ODA, the bending ether linkage inhibits its linking planar segments forming a coplanar conformation, which results in a less ordering structure. Though, some degrees of film orientation still can be distinguished by the presence of a small peak at 5.94 degrees in the in-plane pattern, while no peak presents in the out-of-plane pattern. This peak corresponds to the intramolecular spacing of the repeating monomeric units of PMDA-ODA. ${ }^{21,22}$ The repeat distance is $1.487 \mathrm{~nm}$. The polyimide chains in the on-substrate cured film of PMDA-ODA are slightly preferably aligned in the plane direction.

As can be seen clearly, in the out-of-plane direction, PMDA-PDA and PMDA-B exhibit very different diffraction patterns when comparing with PMDA-ODA. These out-of-plane diffraction patterns, which provide interchain packing information, can then be used to investigate the mixing status of the blends. In case of incompatible or phase segregation, the interchain packing of one polymer will not be inerfered by the other in the system. In case of compatible or phase mixing, ordering owing to interchain packing will be destroyed. These differences can be seen from the intensities of the characteristic diffraction peaks.

\section{Compatibility}

When mixing a highly ordered chain structure polyimide with a much less ordered one, such as PMDA-ODA, one expects to observe a significant reduction in diffraction intensity at its characteristic peak. If the blend system is entirely incompatible, one would expect to see an intensity decrease proportional 
to the composition of PMDA-ODA. In other words, the law of superposition should obey. If the system is compatible, intermixing of regular and irregular chains would drastically reduce the possibility of forming ordered structures. Consequently, the resulted diffraction intensities would be much weaker than expected by the law of superposition.

Figure 6 shows the diffraction peaks of the polyblends of PMDA-ODA with PMDAPDA. The peak height or intensity decreases with the increase of the composition of PMDA-ODA. As can be seen with an addition of $25 \mathrm{wt} \%$ of PMDA-ODA, for example, the peak intensity dropped more than $50 \%$. This change is not in proportion, and nor are those of different compositions. The PMDA-ODA chains do affect the ordered arrangement of the PMDA-PDA. It is noteworthy that all these blends were mixed for five minutes only. It is therefore implied that these blend systems, with PMDA-ODA compositions ranged from 25 to $75 \mathrm{wt} \%$, are compatible.

Actually, beside the ether linkage in the PMDA-ODA structure, the two precursor moleculs have two free rotating single bonds per repeat-unit. These give both polymers a substantially high flexibility in forming random coil-like structures. Ideally speaking, in solution state, there is seldom any reason for two polymers having very similar chemical char-

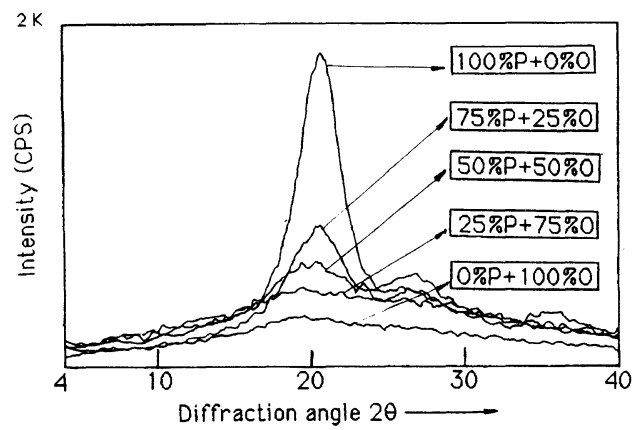

Figure 6. Out-of-plane diffraction patterns of the stacked films of the blends of PMDA-PDA with PMDA-ODA with various compositions. (PMDA-ODA $=\mathrm{O}$; PMDA$\mathrm{PDA}=\mathrm{P}$ ) acteristics, structures and conformations not to be compatible. The blends in the solution state should be compatible. It may be just a matter of mixing time. Of course, the compatible states in solution must be kept very much unaffected upon curing. Otherwise, dissimilarity of the two cured imide chain structures would cause phase separation and turn the systems into incompatible, and the diffraction patterns of the blends would have been different.

Figure 7 shows the diffraction peaks of the polyblends of PMDA-ODA with PMDA-B. The peak height or intensity also decreases with the increase of the composition of PMDAODA. But, the decrease in peak intensity are not so drastic when compared with those in the previous systems. Furthermore, the decreases are closely proportional to the PMDAODA compositions. Apparently, PMDAODA chains do not interfere the formation of the ordered sructures of PMDA-B. Again, these blends were mixed for five minutes only. It is implied that these blend systems are incompatible. The amic acid chain structures of PMDA-PDA and PMDA-B are quite similar to each other. But, one is compatible to PMDA-ODA and the other not. This may be attributed to the differences in their molecular weights and chemical structure. From our observation, the PMDA-B solution as prepared seems slightly more viscous than the MDA-

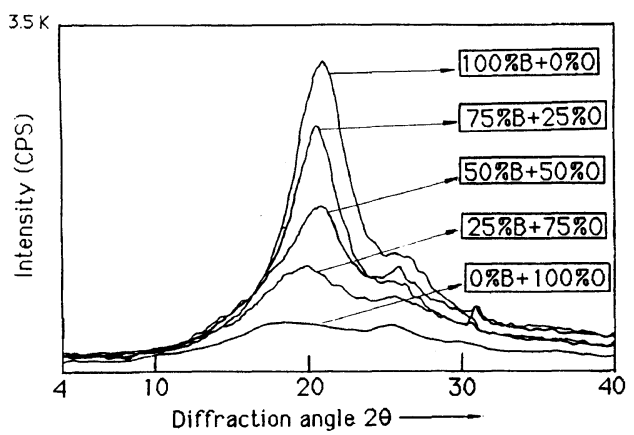

Figure 7. Out-of-plane diffraction patterns of the stacked films of the blends of PMDA-B with PMDA-ODA with various compositions. (PMDA-ODA $=\mathrm{O}$; $\mathrm{PMDA}-\mathrm{B}=\mathrm{B}$ ) 


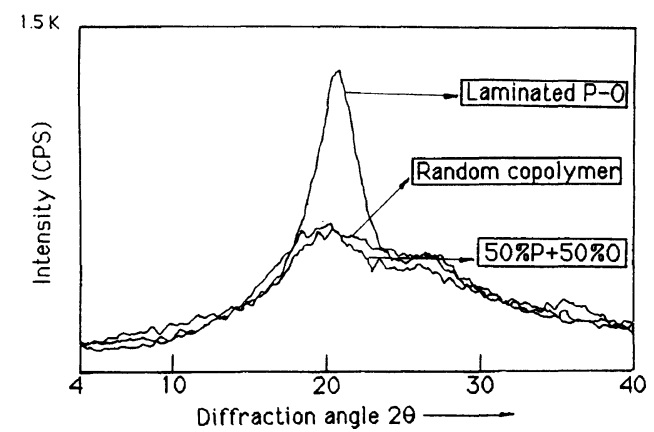

Figure 8. Out-of-plane diffraction patterns of the laminate, blend and random copolyimdes of $50 \mathrm{wt} \%$ PMDA-PDA with $50 \mathrm{wt} \%$ PMDA-ODA. The blend and the random copolyimide have similar patterns.

PDA solution. Since both solutions have the same solid content, it therefore implies that the molecular weight of PMDA-B is somehow higher, relatively speaking. Though, the effect of the molecular weight of the pecursor molecules on the compatibility of the polyimide blends is required further investigation.

Figure 8 compares the out-of-plane diffraction peaks of the polyblend, random copolymer, and laminate with 50\% PMDA-ODA and $50 \%$ PMDA-PDA. The diffraction patterns of the blend and random copolymer are almost not distinguishable. As mentioned in the refernces, ${ }^{8-11}$ polyamic acids in the solution state may undergo exchange reactions with other amic acid compounds. These exchange reactions take place when anhydride and amine functional groups are formed upon chain scission of of amic acid. Binary mixtures of polyamic acids undergone exchange reactions would consequently end up with a compolymer. It is supposed to be a block copolymer initially. Allowed mixed for a long period of time, it would eventually end up with a random copolymer. It is because exchange reactions would further proceed. Furthermore, chain scission reactions can just occur at any suitable sites randomly. Ideally speaking, if the binary mixture in solution is compatible in nature, a random copolymer can be obtained in a much shorter mixing time period than that incompat-

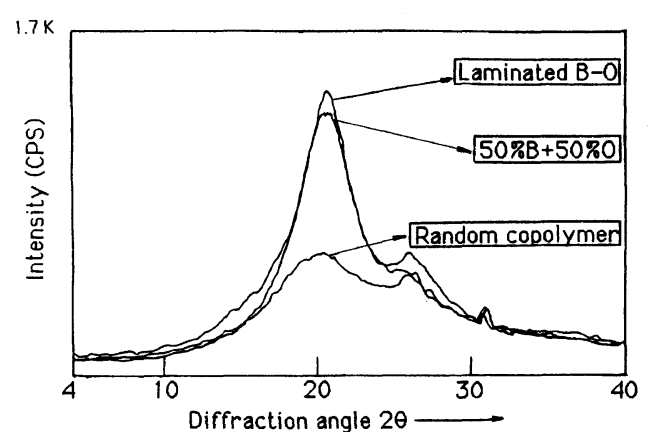

Figure 9. Out-of-plane diffraction patterns of the laminate, blend and random copolyimdes of $50 \mathrm{wt} \%$ PMDA-B with $50 \mathrm{wt} \%$ PMDA-ODA. The pattern of the blend is very different from that of the random copolyimide.

ible. In this blend system, the mixture was mixed for five minutes. From Figure 8 , it is plausible to say that the resulted mixture of the $50 \%$ PMDA-ODA and 50\% PMDA-PDA polyblend has converted to a random copolyamic acid, which has later become a random copolyimide upon thermal curing.

The results for the system with 50\% PMDA$\mathrm{B}$ and 50\% PMDA-ODA are shown in Figure 9. As seen, the blend diffraction pattern is so different from that of the corresponding random copolymer, but so similar to that of the laminate. It indicates that this system is totaly incompatible. Such an incompatibility would change with mixing time if there exist exchange reactions in this system.

\section{Composition and Mixing Time Effects}

From the results given above, it is known that the diffraction intensities of the characteristic peaks are very different with varying compositions. Therefore, same composition must be used in comparison. But, due to the difficulty in preparing these different specimens, especially the laminates, with exactly the same compositions, comparison has been made according to the integrated intensities of the diffraction peaks. Using these data, one can establish for each specimen a characteristic line or curve with respect to its composition. Using these characteristic lines, one can distinguish if 


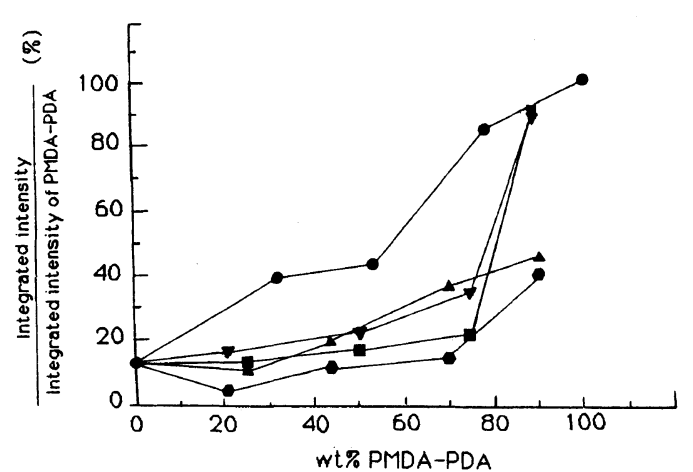

Figure 10. The characteeristic curves of the integrated peak intensities with respect to composition for the (O) laminates, $(\boldsymbol{\nabla})$ blends mixed for $5 \mathrm{~min},(\boldsymbol{\square})$ blends mixed for $5 \mathrm{~min}$ and kept at room temperature overnight, ( $\boldsymbol{\Delta})$ random copolymers, and ( ) alternating copolymers of PMDA-PDA with PMDA-ODA.

the systems are compatible or not at different compositions.

Figure 10 shows the resulted curves representing the peak intensities at vaious compositions for the PMDA-ODA/PMDA-PDA series. Lines $(\boldsymbol{O}),(\boldsymbol{\nabla}),(\boldsymbol{\square}),(\boldsymbol{\Delta})$, and $(\boldsymbol{\bullet})$ are for the laminates, blends mixed for five minutes, blends mixed for five minutes and kept at room temperature overnight, random copolymers, and alternating copolymers, respectively. Within machine resolution, the laminate line, which represents complete phase separation, obeys the law of superposition. As shown, the blend curves are very different from the laminate line, but very similar to the line for the rndom copolymers. It can then be concluded that this blend system is compatible at all compositions except at above $90 \%$ of PMDA-PDA. Furthermore, these compatible blends seem to have a already converted to random copolymers.

As to the alternating copolymers, their characteristic diffraction peaks are the weakest among all samples, as shown in Figure 10. From the standpoint of monomerically mixing scale, no copolymers, except alternating copolymers, have more thorough intermixing status than random copolymers, as generally known. Since the laminates, which typify no

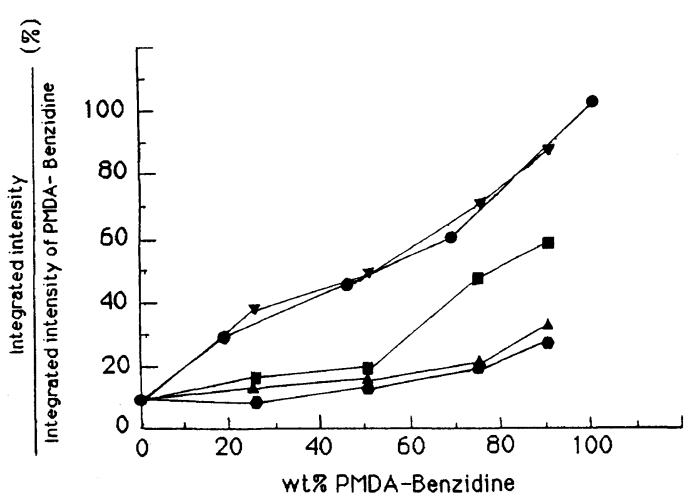

Figure 11. The charactristic curves of the integrated peak intensities with respect to composition for the (O) laminates, $(\boldsymbol{\nabla})$ blends mixed for $5 \mathrm{~min},(\boldsymbol{\square})$ blends mixed for $5 \mathrm{~min}$ and kept at room temperature for two days, $(\boldsymbol{A})$ random copolymers, and ( ) alternating copolymers of PMDA-B with PMDA-ODA.

intermixing, have the strongest diffraction intensities, and the random copolymers, which typify random intermixing from monomeric point of view, have relatively weak diffraction intensities, the presumed alternating copolymers that exhibit even weaker diffraction intensities can be proved be alternating. If these were "block" copolymers, their diffraction intensities should have be, presumably, more or less stronger than those of the random copolymers. This, of course, shall depend on if there are di- or multi-block copolymers. Nevertheless, further examinations are definitely needed to clarify such an argument.

Figure 11 shows the curves for the PMDA-ODA/PMDA-B series. Lines (O), $(\nabla),(\square),(\Delta)$, and $(\boldsymbol{\theta})$ are for the laminates, blends mixed for five minutes, blends mixed for five minutes and kept at room temperature for two days, random copolymers, and alternating copolymers, respectively. As shown, the blend cuve $(\nabla)$ is nearly the same as the laminate line. One can therefore conclude that this blend system is incompatible in nature at all compositions. But, after a much longer mixing time period, i.e. two days, the blends with less PMDA-B content have already become undistinguishable from the corre- 
sponding random copolymers, as indicated by the left part of the blend curve ( $\square$ ). Similar to the results in the PMDA-ODA/PMDA-PDA series, these blends seem to have converted to random copolymers. For the ones with PMDA-B content higher than $70 \%$, their diffraction intensities have also decreased significantly. It is believed that if allowed mixed for an even longer time, these blends would also become completely compatible, or, more likely, become random copolymers. The effect of exchange reactions in these polyimide blends can be seen very prominent, especially when increasing the mixing time.

From this study, one can see that randomization owing to exchange reactions and inherent compatibility both play an important role in determining the final microstructures of the polyimide blends. It seems that the exchange reactions with PMDA-ODA are much faster in PMDA-PDA than in PMDA-B. This may be attributed to the difference in their inherent compatibilities.

\section{CONCLUSIONS}

$\mathrm{X}$-Ray diffraction has been proved in this study a very convenient method in determining polyimide blend compatibility. Several remarks can be summarized as follows.

1. All these three polyimides, PMDAODA, PMDA-PDA and PMDA-B, exhibit film orientation of different extents. The higher in the chain rigidity, the stronger diffraction pattern as well as higher orientation can one observe.

2. The blend systems of PMDA-PDA with PMDA-ODA are compatible at all compositions except above 90\% PMDA-PDA, while those of PMDA-B are not.

3. When kept mixed at room temperature for two days, the blend systems with PMDA-B composition less than $50 \%$, have become undistinguishable from the corresponding random copolymers. For the blends with PMDA-B composition higher than $70 \%$, their diffraction intensities have also decreased significantly.

4. It is plausible to say that the blends with diffraction patterns undistinguishable from those of the corresponding random copolymers have actually converged to random copolymers due to exchange reactions.

5. Randomization owing to the exchange reactions and inherent compatibility both play an important role in determining the final microstructures of th polyimide blends.

6. The exchange reactions with PMDAODA are much faster in PMDA-PDA than in PMDA-B. This may be attributed to the difference in their inherent compatibilities.

Acknowledgement. This work has been supported by the National Science Council, Taiwan, R. O. C. through projects NSC-790405-E007-25 and NSC-78-0401-E007-25.

\section{REFERENCES}

1. P. Burggraaf, Semiconductor International, 58 (1988).

2. R. J. Jenson, IEEE Electronic Components Conference, 1984, p 73.

3. F. K. Moghadam, Solid State Technology, 27, 149 (1984).

4. S. D. Senturia, Proceedings of the 2nd International Conference on Polyimides, 1985, p 107.

5. D. R. Day, Proceddings of the 1st International Conference on Polyimides, 1982, p 767.

6. L. B. Rothman, J. Electrochem. Soc., Solid State Sci., Technol., 127, 2216 (1980).

7. J. H. Jou and T. C. Tsai, NSC Summer Project Report NSC-78-0401-E007-25 (1989).

8. C. Feger, "Polymeric Materials for Electronics Packing and Interconnection and Information," J. H. Lupinski and R. S. Moore, Ed., ACS Polymer Symposirm Series No. 407, 1989, p 114.

9. D. Brekner and C. Feger. J. Polym. Sci., Polym. Chem. Ed., 25, 2479 (1987).

10. M. I. Bessonow, M. M. Koton, V. V. Kudryavtsev, and L. A. Laius, "Polyimides, Thermally Stable Polymers," Consultants Bureau, New York, N.Y., 1987.

11. C. C. Walker, J. Polym. Sci., Polym. Chem., 26, 1649 (1988).

12. M. Ree, D.Y. Yoon, and W. Volksen, Proceedings of the ACS Division of Polymeric Materials: Sci. \& Eng., 60, 179 (1989). 
13. J. H. Jou and P. T. Huang, "1st Pacific Polymer Preprints," Vol. 1, 1989, p 291.

14. J. H. Jou and P. T. Huang, Proceeding of the 11 th ROC Polymer Symposium, 1988.

15. C. E. Sroog, J. Polym. Sci., Macromol. Rev., 11, 161 (1976).

16. I. K. Varma, R. N. Geol, and D. S. Varma, J. Polym. Sci., Polym. Chem. Ed., 17, 5703 (1979).

17. J. Galiani, Chem. Abstr., 91, 159171g (1979).

18. G. N. Babu and S. Samant, Eur. Polym. J., 17, 421
(1981).

19. G. N. Babu and S. Samant, Makromol. Chem., 183, 1129 (1982).

20. J. H. Jou and P. T. Huang, Annual Conference of the Chinese Society of Matrial Science, to be published.

21. R. M. Ikeda, J. Polym. Sci., Polym. Lett. Ed., 4, 353 (1966).

22. S. Isoda, H. Shimada, M. Kochi, and H. Kambe, $J$. Polm. Sci., Polym. Phys. Ed., 19, 1293 (1981). 